\title{
A NOTE ON OPERATORS FIXING COTYPE SUBSPACES OF $C[0,1]$
}

\author{
I. GASPARIS
}

(Communicated by Thomas Schlumprecht)

\begin{abstract}
Let $K$ be a compact, metrizable space. Let $X$ be a closed, linear subspace of $C(K)$ spanned by a normalized weakly null sequence $\left(f_{n}\right)$ such that $\left(\left|f_{n}\right|\right)$ satisfies a lower $q$ estimate on disjoint blocks with positive coefficients for some $1<q<\infty$. It is proved that every $w^{*}$-compact subset of $B_{C(K)^{*}}$ which norms $X$ is non-separable in norm. This provides an alternative proof of Bourgain's result that every $w^{*}$-compact subset of $B_{C(K)}$ * which norms a subspace with non-trivial cotype is non-separable in norm.
\end{abstract}

\section{INTRODUCTION}

The study of fixing properties of operators on $C(K)$ spaces, with $K$ being compact and metrizable, is important to the isomorphic classification of the complemented subspaces of $C(K)$. Rosenthal [12] proved that every operator $T: C(K) \rightarrow$ $C(K)$ whose dual has non-separable range fixes a copy of $C(K)$. The latter means that $T$ becomes an into isomorphism when restricted to a suitable subspace isomorphic to $C(K)$. Rosenthal derived his result by etablishing that for every $w^{*}$-compact subset $\mathcal{M}$ of $B_{C(K)^{*}}$ which is non-separable in norm, there exists a subspace $X$ of $C(K)$ isomorphic to $C(K)$ which is normed by $\mathcal{M}$. That is, there is some $\rho>0$ so that $\sup _{\mu \in \mathcal{M}}\left|\int_{K} f d \mu\right| \geq \rho\|f\|$ for all $f \in X$.

Rosenthal posed the following problems (see e.g. [13]).

Problem 1. Let $T: C(K) \rightarrow C(K)$ fix a copy of a subspace not containing any isomorph of $c_{0}$. Does $T$ fix a copy of $C(K)$ ?

Problem 2. Let $\mathcal{M} \subset B_{C(K)^{*}}$ be $w^{*}$-compact and norming a subspace not containing any isomorph of $c_{0}$. Is $\mathcal{M}$ non-separable in norm?

It is clear that an affirmative answer to the second problem yields an affirmative answer to the first one which, in turn, would imply that a complemented subspace of $C(K)$ with separable dual is $c_{0}$-saturated. Bourgain [4] solved Problem 2 in the affirmative when $\mathcal{M}$ norms a cotype subspace of $C(K)$. The purpose of this article is to provide an alternative proof of Bourgain's result by showing

Theorem 1.1. Let $K$ be a compact, metrizable space. Let $X$ be a closed, linear subspace of $C(K)$ spanned by a normalized weakly null sequence $\left(f_{n}\right)$ such that $\left(\left|f_{n}\right|\right)$ satisfies a lower $q$ estimate on disjoint blocks with positive coefficients for

Received by the editors January 9, 2012 and, in revised form, April 5, 2012 and June 5, 2012. 2010 Mathematics Subject Classification. Primary 46B03; Secondary 47B38.

Key words and phrases. Operators on spaces of continuous functions, lower $q$ estimates.

This research was partially supported by grant ARISTEIA 1082. 
some $1<q<\infty$. Suppose that $\mathcal{M}$ is a $w^{*}$-compact subset of $B_{C(K)^{*}}$ which norms $X$. Then $\mathcal{M}$ is non-separable in norm.

If $\left(f_{n}\right)$ is a normalized weakly null sequence in a $C(K)$ space, then $\left(\left|f_{n}\right|\right)$ is said to satisfy a $C$-lower $q$ estimate on disjoint blocks with positive coefficients provided that there exist $q \in(1, \infty)$ and $C>0$ so that

$$
\left\|\sum_{i=1}^{n} x_{i}\right\| \geq C\left[\sum_{i=1}^{n}\left\|x_{i}\right\|^{q}\right]^{1 / q}
$$

for every $n \in \mathbb{N}$ and every sequence $\left(x_{i}\right)_{i=1}^{n}$ of finitely and disjointly supported blocks of $\left(\left|f_{i}\right|\right)$ having positive coefficients. That is, for all $i \leq n, x_{i}=\sum_{j \in F_{i}} a_{j}\left|f_{j}\right|$, where $a_{j}>0$ for all $j \in F_{i}$, and the $F_{i}$ 's are pairwise disjoint, finite subsets of $\mathbb{N}$.

Remark. Let us say that $c_{0}^{+}$is finitely and disjointly representable in $\left(\left|f_{i}\right|\right)$ if for all $\epsilon>0$ and $n \in \mathbb{N}$ there exist normalized and disjointly supported blocks $x_{1}, \ldots, x_{n}$ of $\left(\left|f_{i}\right|\right)$ having positive coefficients, so that $\left\|\sum_{i=1}^{n} x_{i}\right\| \leq 1+\epsilon$. By mimicking the proof of Theorem 1.f.12 in vol. 2 of [10, one can show that $c_{0}^{+}$is not finitely and disjointly representable in $\left(\left|f_{i}\right|\right)$ if, and only if, $\left(\left|f_{i}\right|\right)$ satisfies a lower $q$ estimate on disjoint blocks with positive coefficients for some $1<q<\infty$.

Theorem 1.1 implies Bourgain's result. Indeed, assume $X$ is a subspace of $C(K)$ with non-trivial cotype $q$ which is normed by a $w^{*}$-compact subset $\mathcal{M}$ of $B_{C(K)^{*}}$. If $X^{*}$ is non-separable, then $\mathcal{M}$ must be non-separable in norm 9 . If $X^{*}$ is separable, then $X$ contains a normalized weakly null sequence $\left(f_{n}\right)$. It is not hard to see then (see Lemma 5 in [4) that every sequence of blocks of $\left(\left|f_{n}\right|\right)$ with positive coefficients (not necessarily disjointly supported) satisfies a lower $q$ estimate. We can thus apply Theorem 1.1 and conclude that $\mathcal{M}$ is non-separable in norm. So formally, when $X^{*}$ is separable, the assumption that $X$ is spanned by a normalized weakly null sequence $\left(f_{n}\right)$ for which $\left(\left|f_{n}\right|\right)$ satisfies a non-trivial lower $q$ estimate on disjoint blocks with positive coefficients is weaker than assuming that $X$ has non-trivial cotype. We do not know, however, of an example which separates these concepts. In fact, we do not even know if $c_{0}$ embeds in $X$ under the assumptions of Theorem 1.1 .

A thorough examination of the proof of Lemma 6 in 4 shows that the arguments of [4] actually work under the assumptions of Theorem 1.1. On the other hand we believe that our arguments yield a simpler proof of Bourgain's result. We shall next give three reasons justifying our belief. The first one is that we do not need to make any reduction to the case of norming sets of positive measures. This is a non-trivial step in the proof given in [4. The second reason involves estimates of the form

$$
\int_{K}\left\|\sum_{i=1}^{n} a_{i} \chi_{F_{i}}(t)\left|f_{i}\right|\right\|^{q} d|\mu|(t) \leq C \delta\left\|\sum_{i=1}^{n} a_{i}\left|f_{i}\right|\right\|,
$$

where the $a_{i}$ 's are non-negative scalars, the $F_{i}$ 's are closed subsets of $K, \mu \in \mathcal{M}$ and $|\mu|\left(F_{i}\right) \leq \delta$ for all $i \leq n$. Such estimates are crucial for establishing the non-separability of $\mathcal{M}$. Lemma 6 in [4] proves this inequality through an intricate argument. In the present proof, in order to prove a similar inequality, we only need an elementary observation, Lemma 2.1, which states that there is some $C>0$ such that if $u$ is a normalized block of $\left(\left|f_{n}\right|\right)$ with positive coefficients, then there exist a finite set $I \subset \mathbb{N}$ and $t_{0} \in K$ so that $\|u \mid I\| \geq 1 / 2$ and $(u \mid J)\left(t_{0}\right) \geq C\|u \mid J\|^{q}$ for all $J \subset I$. 
Finally, in the hard part of the proof given in [4, it is shown that the Szlenk index of $\mathcal{M}$ is uncountable in order to deduce non-separability. In our case we use the Schreier families $\left(S_{\alpha}\right)_{\alpha<\omega_{1}}$ [3] to show that if $\left(\epsilon_{n}\right)$ is a null sequence of positive scalars, then there exists $\lambda>0$ so that every infinite subset $N$ of $\mathbb{N}$ contains a further infinite subset $L, L=\left(l_{i}\right)$, such that $|\mu|\left(\lim \sup _{i}\left[\left|f_{l_{2 i}}\right| \geq \epsilon_{l_{2 i-1}}\right]\right) \geq \lambda$ for some $\mu \in \mathcal{M}$. It then follows by Lemma 3.1 of $[7$ that $\mathcal{M}$ is non-separable in norm. Let us recall here the statement of the aforementioned lemma: If $\mathcal{N}$ is a norm-separable subset of $C(K)^{*},\left(f_{n}\right)$ is a weakly null sequence in $C(K)$ and $\left(\epsilon_{n}\right)$ is a sequence of positive scalars, then given $N \in[\mathbb{N}]$ there exists $M \in[N]$ so that for every $L \in[M]$, $L=\left(l_{n}\right)$, and all $\mu \in \mathcal{N}$ we have that $|\mu|\left(\lim \sup _{n}\left[\left|f_{l_{2 n}}\right| \geq \epsilon_{l_{2 n-1}}\right]\right)=0$. To prove this lemma we first choose a countable, norm-dense subset $\left(\mu_{n}\right)$ of $\mathcal{N}$. By using Ramsey's Theorem and a diagonalization argument, we find $M \in[N]$ so that for every $L \in[M], L=\left(l_{n}\right)$, one has that

$$
\sum_{n=1}^{\infty}\left|\mu_{k}\right|\left(\left[\left|f_{l_{2 n}}\right| \geq \epsilon_{l_{2 n-1}}\right]\right)<\infty, \forall k \in \mathbb{N},
$$

from which the assertion of the lemma clearly follows.

We use standard Banach space facts and terminology as may be found in [10. A useful source of information on $C(K)$ spaces is [13]. Papers [1] and 2] include results on fixing properties of operators on $C(K)$ spaces. The recent paper [5] contains necessary and sufficient conditions on a general operator $T$ in order for $T^{*}$ to have non-separable range.

\section{Proof of Theorem 1.1}

Theorem 1.1 is a consequence of Corollary 2.5 proved at the end of this section. To simplify our arguments we shall assume that $K$ is totally disconnected. The proof of the general case is similar, using partitions of unity. Moreover, when applying Theorem 1.1 to the case of a cotype subspace $X, K$ must be uncountable, and so, in view of Miljutin's theorem [11, there is no loss of generality in assuming that $K$ is a totally disconnected, uncountable compact metric space.

Assume that $X \subset C(K)$ has a normalized weakly null basis $\left(f_{n}\right)$ such that $\left(\left|f_{n}\right|\right)$ satisfies a $C$-lower $q$ estimate on disjoint blocks with positive coefficients. Let $\mathcal{M} \subset B_{C(K)^{*}}$ be $w^{*}$-compact and norming for $X$. By using a standard perturbation argument based on the uniform continuity of the $f_{n}$ 's and the total disconnectivity of $K$, we may assume, without loss of generality, that each $f_{n}$ is a simply continuous function on $K$. That is, there exist finite collections of scalars $\left(b_{n, i}\right)_{i=1}^{k_{n}}$ in $[-1,1]$ and pairwise disjoint, non-empty clopen subsets $\left(W_{n, i}\right)_{i=1}^{k_{n}}$ of $K$ so that $f_{n}=\sum_{i=1}^{k_{n}} b_{n, i} \chi_{W_{n, i}}$. Given $\mu \in \mathcal{M}$ and $\epsilon>0$, set

$$
\mu\left(f_{n}, \epsilon\right)=\sum_{i:\left|b_{n, i}\right| \geq \epsilon}\left|\mu\left(W_{n, i}\right)\right| .
$$

Clearly, $0 \leq \mu\left(f_{n}, \epsilon\right) \leq|\mu|\left[\left|f_{n}\right| \geq \epsilon\right] \leq\|\mu\| \leq 1$. It is easy to see that if $0<\epsilon<\delta$, then

$$
\left|\int_{\left[\epsilon \leq\left|f_{n}\right|<\delta\right]} f_{n} d \mu\right| \leq \delta \mu\left(f_{n}, \epsilon\right) .
$$


Lemma 2.1. Let $u=\sum_{i \in F} a_{i}\left|f_{i}\right|$, where $F$ is a finite subset of $\mathbb{N}$ and $\left(a_{i}\right)_{i \in F}$ are positive scalars. Suppose that $\|u\|=1$. Then there exist $t_{0} \in K$ and $I \subset F$ so that:

(1) $\|u \mid I\| \geq 1 / 2$,

(2) $(u \mid J)\left(t_{0}\right) \geq\left(C^{q} / 2\right)\|u \mid J\|^{q}$ for all $J \subset I$.

Proof. Choose $t_{0} \in K$ so that $u\left(t_{0}\right)=1$. Let $\left\{F_{1}, \ldots, F_{l}\right\}$ be a maximal family of pairwise disjoint subsets of $F$ so that

$$
\left(u \mid F_{i}\right)\left(t_{0}\right)<\left(C^{q} / 2\right)\left\|u \mid F_{i}\right\|^{q}, \forall i \leq l .
$$

Set $I=F \backslash \bigcup_{i=1}^{l} F_{i}$. Then,

$$
\begin{aligned}
(u \mid F \backslash I)\left(t_{0}\right) & <\left(C^{q} / 2\right) \sum_{i=1}^{l}\left\|u \mid F_{i}\right\|^{q} \\
& \leq\left(C^{q} / 2\right)\left(1 / C^{q}\right)\|u \mid F \backslash I\|^{q} \leq 1 / 2 .
\end{aligned}
$$

Therefore, $(u \mid I)\left(t_{0}\right) \geq 1 / 2$, and so by maximality $I$ is as desired.

Lemma 2.2. Let $N \in[\mathbb{N}], \alpha<\omega_{1}$ and $\epsilon>0$. There exist a finite set $I \subset N$ and positive scalars $\left(a_{i}\right)_{i \in I}$ so that

$$
\left\|\sum_{i \in I} a_{i}\left|f_{i}\right|\right\|=1 \text { and }\left\|\sum_{i \in J} a_{i}\left|f_{i}\right|\right\|<\epsilon, \forall J \subset I, J \in S_{\alpha} .
$$

The proof of this lemma is easily done by transfinite induction using the following consequence of the lower $q$ estimate on disjoint blocks of $\left(\left|f_{n}\right|\right)$ with positive coefficients: $\sup _{n}\left\|\sum_{i=1}^{n} u_{i}\right\|=\infty$ for every normalized block basis $\left(u_{n}\right)$ of $\left(\left|f_{n}\right|\right)$ with positive coefficients. We refer to 8 for details.

In our next proposition we use an idea from [4], namely, to find an upper estimate for $\int_{K}\left\|\sum_{i=1}^{n} a_{i} \chi_{F_{i}}(t)\left|f_{i}\right|\right\|^{q} d|\mu|(t)$, where $F_{i}=\left[\left|f_{i}\right| \geq D|\mu|\left(\left|f_{i}\right|\right)\right], i \leq n$, and $D>0$. Lemma 2.1 will be very useful in this task.

Proposition 2.3. Assume that $\mathcal{M} \rho$-norms $X$ for some $\rho>0$. Let $\left(\epsilon_{n}\right)$ be a null sequence of positive scalars. Then there exists $\lambda>0$ depending only on $\rho, q$ and $C$ so that the following property is satisfied: For every $\alpha<\omega_{1}$ and every infinite subset $N$ of $\mathbb{N}, N=\left(n_{i}\right)$, there exist $\mu \in \mathcal{M}$ and a finite subset $I$ of $\mathbb{N}$ so that

(1) $\mu\left(f_{n_{2 i}}, \epsilon_{n_{2 i-1}}\right) \geq \lambda$ for all $i \in I$,

(2) $\left\{n_{2 i}: i \in I\right\} \notin S_{\alpha}$.

Proof. We may clearly assume that $\sum_{n \in N} \epsilon_{n}<\rho / 4$. Let $\epsilon>0$ be small enough to be determined later. Lemma 2.2 yields a finite set $I_{1} \subset \mathbb{N}$ and positive scalars $\left(a_{i}\right)_{i \in I_{1}}$ so that

$$
\left\|\sum_{i \in I_{1}} a_{i}\left|f_{n_{2 i}}\right|\right\|=1 \text { and }\left\|\sum_{i \in J} a_{i}\left|f_{n_{2 i}}\right|\right\|<\epsilon, \forall J \subset I_{1},\left\{n_{2 i}: i \in J\right\} \in S_{\alpha} .
$$

Apply Lemma 2.1 to obtain $I_{2} \subset I_{1}$ and $t_{0} \in K$ so that $\left\|\sum_{i \in I_{2}} a_{i}\left|f_{n_{2 i}}\right|\right\| \geq 1 / 2$ and

$$
\left\|\sum_{i \in J} a_{i}\left|f_{n_{2 i}}\right|\right\|^{q} \leq\left(2 / C^{q}\right) \sum_{i \in J} a_{i}\left|f_{n_{2 i}}\left(t_{0}\right)\right|, \forall J \subset I_{2} .
$$

We next choose signs $\left(\sigma_{i}\right)_{i \in I_{2}}$ so that $\left\|\sum_{i \in I_{2}} a_{i}\left|f_{n_{2 i}}\right|\right\|=\left\|\sum_{i \in I_{2}} a_{i} \sigma_{i} f_{n_{2 i}}\right\|$. 
Pick $\mu \in \mathcal{M}$ so that $\left|\int_{K} \sum_{i \in I_{2}} a_{i} \sigma_{i} f_{n_{2 i}} d \mu\right| \geq \rho / 2$.

Put $\delta_{i}=\int_{K}\left|f_{n_{2 i}}\right| d|\mu|$ for all $i \in I_{2}$ and set $I_{0}=\left\{i \in I_{2}: \delta_{i}>0\right\}$. Let $D>0$ to be determined later, and define

$$
\phi_{i}=\chi_{\left[\left|f_{n_{2 i}}\right| \geq D \delta_{i}\right]}, \forall i \in I_{0} .
$$

Of course, $\phi_{i}$ is upper semicontinuous for all $i \in I_{0}$. It is clear that

$$
\rho / 2 \leq\left|\int_{K} \sum_{i \in I_{0}} a_{i} \sigma_{i} f_{n_{2 i}} \phi_{i} d \mu\right|+\left|\int_{K} \sum_{i \in I_{0}} a_{i} \sigma_{i} f_{n_{2 i}}\left(1-\phi_{i}\right) d \mu\right| .
$$

Let $A=\int_{K} \sum_{i \in I_{0}} a_{i} \sigma_{i} f_{n_{2 i}} \phi_{i} d \mu$ and $B=\int_{K} \sum_{i \in I_{0}} a_{i} \sigma_{i} f_{n_{2 i}}\left(1-\phi_{i}\right) d \mu$. We first estimate

$$
\begin{aligned}
|B| & \leq \sum_{i \in I_{0}} a_{i}\left|\int_{\left[\left|f_{n_{2 i}}\right|<D \delta_{i}\right]} f_{n_{2 i}} d \mu\right| \\
& \leq \sum_{i \in I_{0}} a_{i}\left|\int_{\left[\epsilon_{n_{2 i-1}} \leq\left|f_{n_{2 i}}\right|<D \delta_{i}\right]} f_{n_{2 i}} d \mu\right|+\sum_{i \in I_{0}} a_{i} \int_{\left[\left|f_{n_{2 i}}\right|<\epsilon_{n_{2 i-1}}\right]}\left|f_{n_{2 i}}\right| d|\mu| \\
& \leq D \sum_{i \in I_{0}} a_{i} \delta_{i} \mu\left(f_{n_{2 i}}, \epsilon_{n_{2 i-1}}\right)+\sum_{i \in I_{0}} a_{i} \epsilon_{n_{2 i-1}} \text {, by (2.1) } \\
& \leq D \sum_{i \in I_{0}} a_{i} \delta_{i} \mu\left(f_{n_{2 i}}, \epsilon_{n_{2 i-1}}\right)+\rho / 4
\end{aligned}
$$

We next estimate

$$
\begin{aligned}
|A| & \leq \int_{K} \sum_{i \in I_{0}} a_{i} \phi_{i}\left|f_{n_{2 i}}\right| d|\mu| \\
& \leq \int_{K} \| \sum_{i \in I_{0}} a_{i} \phi_{i}(t)\left|f_{n_{2 i}}\right||d| \mu \mid(t) \\
& \leq\left[\int_{K} \|\left.\sum_{i \in I_{0}} a_{i} \phi_{i}(t)\left|f_{n_{2 i}}\right|\right|^{q} d|\mu|(t)\right]^{1 / q}, \text { by Jensen's inequality } \\
& \leq\left[\int_{K}\left(2 / C^{q}\right) \sum_{i \in I_{0}} a_{i} \phi_{i}(t)\left|f_{n_{2 i}}\left(t_{0}\right)\right| d|\mu|(t)\right]^{1 / q}, \text { by (2.3) } \\
& \leq\left(2^{1 / q} / C\right)\left[\sum_{i \in I_{0}} a_{i}\left|f_{n_{2 i}}\left(t_{0}\right)\right| \int_{K} \phi_{i} d|\mu|\right]^{1 / q} \\
& \leq\left(2^{1 / q} / C\right)\left[\sum_{i \in I_{0}} a_{i}\left|f_{n_{2 i}}\left(t_{0}\right)\right|(1 / D)\right]^{1 / q} \leq(1 / C)(2 / D)^{1 / q}
\end{aligned}
$$

since $|\mu|\left[\left|f_{n_{2 i}}\right| \geq D \delta_{i}\right] \leq 1 / D$, by Chebyshev's inequality. By taking into account estimates (2.5) and (2.6), (2.4) leads us to

$$
\rho / 4 \leq(1 / C)(2 / D)^{1 / q}+D \sum_{i \in I_{0}} a_{i} \delta_{i} \mu\left(f_{n_{2 i}}, \epsilon_{n_{2 i-1}}\right) .
$$

We conclude that if $(1 / C)(2 / D)^{1 / q}<\rho / 4$, then there exists $\lambda>0$ depending only on $\rho, C$ and $q$ so that

$$
\sum_{i \in I_{0}} a_{i} \delta_{i} \mu\left(f_{n_{2 i}}, \epsilon_{n_{2 i-1}}\right)>2 \lambda
$$


Let $I=\left\{i \in I_{0}: \mu\left(f_{n_{2 i}}, \epsilon_{n_{2 i-1}}\right) \geq \lambda\right\}$. It follows that $\left\{n_{2 i}: i \in I\right\} \notin S_{\alpha}$, since otherwise

$$
\begin{aligned}
2 \lambda & \leq \sum_{i \in I} a_{i} \delta_{i} \mu\left(f_{n_{2 i}}, \epsilon_{n_{2 i-1}}\right)+\sum_{i \in I_{0} \backslash I} a_{i} \delta_{i} \mu\left(f_{n_{2 i}}, \epsilon_{n_{2 i-1}}\right) \\
& \leq \sum_{i \in I} a_{i} \delta_{i}+\lambda, \text { as } \sum_{i \in I_{0}} a_{i} \delta_{i}=|\mu|\left(\sum_{i \in I} a_{i}\left|f_{n_{2 i}}\right|\right) \leq 1 \\
& \leq\left\|\sum_{i \in I} a_{i}\left|f_{n_{2 i}}\right|\right\|+\lambda \leq \epsilon+\lambda, \text { by (2.2), }
\end{aligned}
$$

which yields a contradiction when $0<\epsilon<\lambda$.

Corollary 2.4. Let $X$ be a subspace of $C(K)$ having a normalized weakly null basis $\left(f_{n}\right)$ so that $\left(\left|f_{n}\right|\right)$ satisfies a $C$-lower $q$ estimate on disjoint blocks with positive coefficients. Assume that $\mathcal{M} \subset B_{C(K)^{*}}$ is $w^{*}$-compact and that it $\rho$-norms $X$ for some $\rho>0$. Let $\left(\epsilon_{n}\right)$ be a null sequence of positive scalars. Then there exists $\lambda>0$, depending only on $\rho, q$ and $C$, so that for every $\alpha<\omega_{1}$ and every $N \in[\mathbb{N}]$, $N=\left(n_{i}\right)$, the family

$$
\mathcal{F}_{N}=\left\{\left\{n_{2 i}: i \in I\right\}: I \in[\mathbb{N}]^{<\infty}, \exists \mu \in \mathcal{M}, \mu\left(f_{n_{2 i}}, \epsilon_{n_{2 i-1}}\right) \geq \lambda, \forall i \in I\right\}
$$

is not compact in the topology of pointwise convergence.

Proof. It follows directly from Proposition 2.3 that $\mathcal{F}_{N}$ is contained in $S_{\alpha}$ for no $\alpha<\omega_{1}$. The non-compactness of $\mathcal{F}_{N}$ follows by employing an argument similar to Corollary 5.2 in 6 .

Corollary 2.5. Let $X$ be a subspace of $C(K)$ having a normalized weakly null basis $\left(f_{n}\right)$ so that $\left(\left|f_{n}\right|\right)$ satisfies a $C$-lower $q$ estimate on disjoint blocks with positive coefficients. Assume that $\mathcal{M} \subset B_{C(K)^{*}}$ is $w^{*}$-compact and that it $\rho$-norms $X$ for some $\rho>0$. Let $\left(\epsilon_{n}\right)$ be a null sequence of positive scalars. Then there exists $\lambda>0$, depending only on $\rho, q$ and $C$, so that for every $N \in[\mathbb{N}]$ there exist $L \in[N]$, $L=\left(l_{i}\right)$, and $\mu \in \mathcal{M}$ so that

$$
|\mu|\left[\left|f_{l_{2 i}}\right| \geq \epsilon_{l_{2 i-1}}\right] \geq \lambda, \forall i \in \mathbb{N} .
$$

In particular, $\mathcal{M}$ is non-separable in norm.

Proof. $\mathcal{F}_{N}$ is not pointwise compact thanks to Corollary 2.4 We may thus find $J \in[\mathbb{N}], J=\left(j_{i}\right)$, so that for every $k \in \mathbb{N}$ there exists $\mu_{k} \in \mathcal{M}$ such that

$$
\mu_{k}\left(f_{n_{2 j_{i}}}, \epsilon_{n_{2 j_{i}-1}}\right) \geq \lambda, \forall i \leq k .
$$

Define $l_{2 i}=n_{2 j_{i}}$ and $l_{2 i-1}=n_{2 j_{i}-1}$ for all $i \in \mathbb{N}$ and set $L=\left(l_{i}\right)$. If $\mu \in \mathcal{M}$ is a $w^{*}$-cluster point of $\left(\mu_{k}\right)$ then clearly, $\mu\left(f_{l_{2 i}}, \epsilon_{l_{2 i-1}}\right) \geq \lambda$ for all $i \in \mathbb{N}$. It follows now that

$$
|\mu|\left[\left|f_{l_{2 i}}\right| \geq \epsilon_{l_{2 i-1}}\right] \geq \lambda, \forall i \in \mathbb{N},
$$

and therefore $|\mu|\left(\lim \sup _{i}\left[\left|f_{l_{2 i}}\right| \geq \epsilon_{l_{2 i-1}}\right]\right) \geq \lambda$. Applying Lemma 3.1 of [] we conclude that $\mathcal{M}$ is non-separable in norm. 


\section{REFERENCES}

[1] Dale E. Alspach, Quotients of $C[0,1]$ with separable dual, Israel J. Math. 29 (1978), no. 4, 361-384, DOI 10.1007/BF02761174. MR491925 (80b:46039)

[2] Dale E. Alspach, $C(K)$ norming subsets of $C[0,1]$, Studia Math. 70 (1981), no. 1, $27-61$. MR646959 (83h:46032)

[3] Dale E. Alspach and Spiros Argyros, Complexity of weakly null sequences, Dissertationes Math. (Rozprawy Mat.) 321 (1992), 44. MR1191024 (93j:46014)

[4] J. Bourgain, A result on operators on $\mathcal{C}[0,1]$, J. Operator Theory 3 (1980), no. 2, 275-289. MR.578944(82c:46024)

[5] Pandelis Dodos, Operators whose dual has non-separable range, J. Funct. Anal. 260 (2011), no. 5, 1285-1303, DOI 10.1016/j.jfa.2010.12.004. MR2749429 (2012h:46024)

[6] I. Gasparis, Operators on $C[0,1]$ preserving copies of asymptotic $l_{1}$ spaces, Math. Ann. 333 (2005), no. 4, 831-858, DOI 10.1007/s00208-005-0702-y. MR2195147(2007a:46006)

[7] I. Gasparis, On a problem of H. P. Rosenthal concerning operators on $C[0,1]$, Adv. Math. 218 (2008), no. 5, 1512-1525, DOI 10.1016/j.aim.2008.03.015. MR2419931 (2009e:46011)

[8] I. Gasparis, E. Odell, and B. Wahl, Weakly null sequences in the Banach space C $(K)$, Methods in Banach space theory, London Math. Soc. Lecture Note Ser., vol. 337, Cambridge Univ. Press, Cambridge, 2006, pp. 97-131, DOI 10.1017/CBO9780511721366.005. MR2326381 (2008k:46035)

[9] Richard Haydon, An extreme point criterion for separability of a dual Banach space, and a new proof of a theorem of Corson, Quart. J. Math. Oxford (2) 27 (1976), no. 107, 379-385. MR0493264 (58 \#12293)

[10] Joram Lindenstrauss and Lior Tzafriri, Classical Banach spaces. I. Sequence spaces, Ergebnisse der Mathematik und ihrer Grenzgebiete, Vol. 92, Springer-Verlag, Berlin, 1977. MR0500056 (58 \#17766)

[11] A. A. Miljutin, Isomorphism of the spaces of continuous functions over compact sets of the cardinality of the continuum (Russian), Teor. Funkciǔ Funkcional. Anal. i Priložen. Vyp. 2 (1966), 150-156 (1 foldout). MR0206695 (34 \#6513)

[12] Haskell P. Rosenthal, On factors of $C([0,1])$ with non-separable dual, Proceedings of the International Symposium on Partial Differential Equations and the Geometry of Normed Linear Spaces (Jerusalem, 1972), Israel J. Math. 13 (1972), 361-378 (1973); correction, ibid. 21 (1975), no. 1, 93-94. MR0388063 (52 \#8900)

[13] Haskell P. Rosenthal, The Banach spaces $C(K)$, Handbook of the geometry of Banach spaces, Vol. 2, North-Holland, Amsterdam, 2003, pp. 1547-1602, DOI 10.1016/S1874-5849(03)800438. MR1999603 (2004g:46028)

Department of Mathematics, Aristotle University of Thessaloniki, Thessaloniki, 54124, GREECE

Current address: Department of Mathematics, School of Applied Mathematical and Physical Sciences, National Technical University of Athens, Heroon Polytechneiou 9, Athens, 15780, Greece E-mail address: ioagaspa@math.ntua.gr 